\title{
THE CLOSED RANGE PROPERTY FOR BANACH SPACE OPERATORS
}

\author{
THOMAS L. MILLER \\ Dept. of Mathematics and Statistics, Mississippi State University, Drawer MA, \\ Mississippi State, MS 39762 \\ e-mail:miller@math.msstate.edu \\ and VLADIMIR MÜLLER \\ Mathematical Institute, Czech Academy of Sciences, Zitna 25, 11567 Prague 1, Czech Republic \\ e-mail:muller@math.cas.cz
}

(Received 7 April, 2006; revised 20 February, 2007, 23 July, 2007, 8 August, 2007; accepted 25 August, 2007)

Abstract. Let $T$ be a bounded operator on a complex Banach space $X$. Let $V$ be an open subset of the complex plane. We give a condition sufficient for the mapping $f(z) \mapsto(T-z) f(z)$ to have closed range in the Fréchet space $H(V, X)$ of analytic $X$ valued functions on $V$. Moreover, we show that there is a largest open set $U$ for which the map $f(z) \mapsto(T-z) f(z)$ has closed range in $H(V, X)$ for all $V \subseteq U$. Finally, we establish analogous results in the setting of the weak-* topology on $H\left(V, X^{*}\right)$.

2000 Mathematics Subject Classification. 47A11.

Introduction. Let $X$ be a complex Banach space and denote by $B(X)$ the algebra of bounded linear operators on $X$. For $T \in B(X)$, let $\sigma(T)$ denote the spectrum of $T$, and denote by Lat $(T)$ the collection of closed $T$-invariant subspaces of $X$. If $M \in \operatorname{Lat}(T)$, we write the restriction of $T$ to $M$ as $\left.T\right|_{M}$.

A basic notion in local spectral theory is that of decomposability. Given an open subset $U$ of the complex plane $\mathbb{C}, T \in B(X)$ is said to be decomposable on $U$ provided that for any open cover $\left\{V_{1}, \ldots, V_{n}\right\}$ of $\mathbb{C}$ with $\mathbb{C} \backslash U \subset V_{1}$, there exists $\left\{X_{1}, \ldots, X_{n}\right\} \subset$ Lat $(T)$ such that $X=X_{1}+\cdots+X_{n}$ and $\sigma\left(\left.T\right|_{X_{k}}\right) \subset V_{k}$ for each $k, 1 \leq k \leq n$; see [2], [5], [8], [11], and [12]. That for each $T \in B(X)$ there exists a largest open set $U$ on which $T$ is decomposable was first shown by Nagy, [11].

An alternative characterization of decomposability may be given in terms of a property introduced by E. Bishop, [3]. For an open subset $V$ of $\mathbb{C}$, let $H(V, X)$ denote the space of all analytic $X$-valued functions on $V$. Then $H(V, X)$ is a Fréchet space with generating semi-norms given by $p_{K}(f):=\sup \{\|f(\lambda)\|: \lambda \in K\}$, where $K$ runs through the compact subsets of $V$. Every operator $T \in B(X)$ induces a continuous linear mapping $T_{V}$ on $H(V, X)$, defined by $T_{V} f(\lambda):=(T-\lambda) f(\lambda)$ for all $f \in H(V, X)$ and $\lambda \in V$. An operator $T$ is said to possess Bishop's property $(\beta)$ on an open set $U \subset \mathbb{C}$ if for each open subset $V$ of $U$, the operator $T_{V}$ is injective with range $\operatorname{ran} T_{V}$

\footnotetext{
Part of this work has been prepared while the first author was a guest of the Mathematical Institute of the Czech Academy of Sciences. He would like to express his gratitude to the Institute and to his coauthor for his hospitality. The second author was supported by grant No. 201/06/0128 of GA ČR and by Institutional Research Plan AV 0Z 10190503.
} 
closed in $H(V, X)$; see [6, Prop. 1.2.6]. Clearly there exists a largest open set $\rho_{\beta}(T)$ on which $T$ has property $(\beta)$.

Fundamental work by Albrecht and Eschmeier established that an operator $T \in$ $B(X)$ has property $(\beta)$ on $U$ precisely when there exists an operator $S \in B(Y)$ such that $S$ is decomposable on $U, X \in \operatorname{Lat}(S)$ and $T=\left.S\right|_{X}$, [2, Theorem 10]. Moreover, [2, Theorems 8 and 21], $T$ is decomposable on $U$ if and only if $T$ and its adjoint $T^{*}$ share property $(\beta)$ on $U$. Thus Nagy's largest open set on which $T$ is decomposable is the set $\rho_{\beta}(T) \cap \rho_{\beta}\left(T^{*}\right)$.

An operator $T \in B(X)$ is said to have the single-valued extension property (SVEP) at a point $\lambda \in \mathbb{C}$ provided that, for every open disc $V$ centered at $\lambda$, the mapping $T_{V}$ is injective on $H(V, X)$. If $U \subset \mathbb{C}$ is open, then $T$ is said to have SVEP on $U$ if $T$ has SVEP at every $\lambda \in U$, equivalently, if $T_{V}$ is injective for each open set $V \subseteq U$. Let $\rho_{\mathrm{SVEP}}(T)$ denote the largest open set on which $T$ has SVEP.

Recently, M. Neumann, V. Miller and the first author of the current paper showed, [9, Theorem 2.5], that $T_{V}$ has closed range in $H(V, X)$ for every open subset $V$ of the "Kato-type" resolvent set of $T$, an open set that contains the semi-Fredholm region of $T$, thus extending a result of Eschmeier, [5]. Following Neumann, we say that an operator has the closed range property $(\mathrm{CR})$ on an open set $U \subset \mathbb{C}$ provided $\operatorname{ran}\left(T_{V}\right)$ is closed in $H(V, X)$ for every open subset $V$ of $U$. Thus $T$ has property $(\beta)$ on $U$ if and only if $T$ has both SVEP and (CR) on $U$.

In this note, we give a more general condition that suffices for $T \in B(X)$ to have (CR) on an open set $U$ and prove that there is in fact a largest open set $\rho_{\mathrm{CR}}(T)$ on which $T$ has the closed range property. Thus $\rho_{\beta}(T)=\rho_{\mathrm{SVEP}}(T) \cap \rho_{\mathrm{CR}}(T)$. In the last section we establish corresponding results in the setting of the weak-* topology on $H\left(V, X^{*}\right)$.

Main results. We denote the kernel of $T \in B(X)$ by $\operatorname{ker}(T)$ and define $N^{\infty}(T):=$ $\bigcup_{n \geq 0} \operatorname{ker}\left(T^{n}\right)$ and $R^{\infty}(T):=\bigcup_{n \geq 0} \operatorname{ran}\left(T^{n}\right)$. If $T \in B(X)$ is such that $\operatorname{ran}(T)$ is closed and $N^{\infty}(T) \subseteq R^{\infty}(T)$, then $T$ is said to be a Kato operator. A systematic exposition of this class, also referred to as semi-regular operators, may be found in [10, Section II.12]; also see [1, Section 1.2] and [6, Section 3.1]. In particular, an equivalent condition may be given in terms of the reduced minimum modulus function: for $S \in B(X)$, define $\gamma(S):=\inf \{\|S x\|: \operatorname{dist}(x, \operatorname{ker}(S))=1\}$. Then an operator $T$ is Kato if and only if $\gamma(T)>0$ and the mapping $z \rightarrow \gamma(T-z)$ is continuous at 0 , [10, II.12 Theorem 2]. Denote by $\sigma_{K}(T)$ the set of all $\lambda \in \mathbb{C}$ such that $T-\lambda$ is not Kato. Then $\sigma_{K}(T)$ is a nonempty compact set, $z \mapsto R^{\infty}(T-z)$ is constant on each component of $\rho_{K}(T):=$ $\mathbb{C} \backslash \sigma_{K}(T), R^{\infty}(T-\lambda)$ is closed and $(T-\lambda) R^{\infty}(T-\lambda)=R^{\infty}(T-\lambda)$ for each $\lambda \in$ $\rho_{K}(T)$, [10, II.12, Theorem 15 and Cor. 19]. Moreover, if $G$ is a component of $\rho_{K}(T)$ and $S \subset G$ has an accumulation point in $G$, then $\bigcap_{z \in S} \operatorname{ran}(T-z)=R^{\infty}(T-\lambda)$ for each $\lambda \in G,[\mathbf{6}, 3.1 .11]$.

For each closed subset $F$ of $\mathbb{C}$, define the "glocal" analytic spectral subspace $\mathfrak{X}_{T}(F):=\left\{x \in X: x \in \operatorname{ran} T_{\mathbb{C} \backslash F}\right\}$. These spaces are $T$-invariant, but generally not closed. If $M \in \operatorname{Lat}(T)$ and $V \subset \mathbb{C}$ is such that $(T-z) M=M$ for all $z \in V$, then $M \subset \mathfrak{X}_{T}(\mathbb{C} \backslash V)$ by a theorem of Leiterer, [6, Theorem 3.2.1]. It follows from above that if $G$ is a component of $\rho_{K}(T)$ and $V \subset G$ is open, then $\mathfrak{X}_{T}(\mathbb{C} \backslash V)=R^{\infty}(T-\lambda)$ for all $\lambda \in G$; in particular, $\mathfrak{X}_{T}(\mathbb{C} \backslash V)$ is closed. Also, it is easily seen that if $T$ has (CR) on an open set $U$, then $\mathfrak{X}_{T}(\mathbb{C} \backslash V)$ is closed for every open $V \subset U$.

A consequence of Theorem 5 below is that the converse holds under the additional assumption that $\operatorname{ran}(T-z)$ is closed for all but countably many $z \in V$. Some additional 
assumption beyond closeness of the glocal spectral subspaces is seen to be necessary for (CR) by the facts that, on one hand, $T$ has property $(\beta)$ on all of $\mathbb{C}$ precisely when $T$ has $(\mathrm{CR})$ on $\mathbb{C}$, [6, Prop. 3.3.5], while on the other hand, there is an operator $T \in B(X)$ without property $(\beta)$ but for which $\mathfrak{X}_{T}(F)$ is closed for all closed $F \subset \mathbb{C}$, [7].

If $(X, d)$ is a metric space, let $B(x, r)$ denote the open ball in $X$ with radius $r>0$ and center $x \in X$.

Lemma 1. Let $T \in B(X)$ and let $V$ be an open subset of $\mathbb{C}$. Let $\left(D_{i}\right)_{i \in A}$ be a cover of $V$ consisting of simply connected open sets $D_{i}$ such that $\mathfrak{X}_{T}\left(\mathbb{C} \backslash D_{i}\right)$ is closed for each $i \in A$ and $D_{i} \backslash D_{j} \neq \emptyset$ if $\mathfrak{X}_{T}\left(\mathbb{C} \backslash D_{i}\right) \neq \mathfrak{X}_{T}\left(\mathbb{C} \backslash D_{j}\right)$.

Let $M=\bigcap_{i \in A} \mathfrak{X}_{T}\left(\mathbb{C} \backslash D_{i}\right)$. Then $M$ is closed, $T M \subset M$ and

(i) if $x \in M$ and $g_{j} \in H\left(D_{j}, X\right)$ are such that $T_{D_{j}} g_{j}=x$, then $g_{j}\left(D_{j}\right) \subset M$;

(ii) $\operatorname{ker} T_{D_{j}} \subset H\left(D_{j}, M\right)$;

(iii) $(T-z) M=M$ for all $z \in V$;

(iv) if $\widetilde{T}: X / M \rightarrow X / M$ is the quotient map induced by $T$ then $\widetilde{T}_{D_{j}}$ is injective on $H\left(D_{j}, X / M\right)$.

Proof. Clearly $M$ is a closed subspace of $X$ and $T M \subset M$.

(i) Let $x \in M$ and $g_{j} \in H\left(D_{j}, X\right)$ be such that $T_{D_{j}} g_{j}=x$.

We show first that $g_{j}\left(D_{j}\right) \subset \mathfrak{X}_{T}\left(\mathbb{C} \backslash D_{j}\right)$. Let $z \in D_{j}$, and define $h_{j}: D_{j} \rightarrow X$ by $h_{j}(\omega)=$ $\left(g_{j}(\omega)-g_{j}(z)\right) /(\omega-z)$ if $\omega \in D_{j} \backslash\{z\}$ and $h_{j}(z)=g_{j}^{\prime}(z)$. Then $h_{j} \in H\left(D_{j}, X\right)$ and if $\omega \neq$ $z$, then

$$
(T-\omega) h_{j}(\omega)=\frac{1}{\omega-z}\left(x-((T-z)+(z-\omega)) g_{j}(z)\right)=g_{j}(z)
$$

By continuity, $(T-z) h_{j}(z)=g_{j}(z)$ as well. Hence $g_{j}(z) \in \mathfrak{X}_{T}\left(\mathbb{C} \backslash D_{j}\right)$ and so $g_{j}\left(D_{j}\right) \subset$ $\mathfrak{X}_{T}\left(\mathbb{C} \backslash D_{j}\right)$.

If $i \in A$ is such that $\mathfrak{X}_{T}\left(\mathbb{C} \backslash D_{i}\right) \neq \mathfrak{X}_{T}\left(\mathbb{C} \backslash D_{j}\right)$, let $g_{i} \in H\left(D_{i}, X\right)$ be such that $T_{D_{i}} g_{i}=x$, let $z \in D_{j} \backslash D_{i}$ and define $h_{i}: D_{i} \rightarrow X$ by $h_{i}(\omega)=\left(g_{i}(\omega)-g_{j}(z)\right) /(\omega-z)$. Then $h_{i} \in H\left(D_{i}, X\right)$ and again

$$
\begin{aligned}
(T-\omega) h_{i}(\omega) & =\frac{1}{\omega-z}\left((T-\omega) g_{i}(\omega)-((T-z)+(z-\omega)) g_{j}(z)\right) \\
& =\frac{1}{\omega-z}\left(x-x+(\omega-z) g_{j}(z)\right)=g_{j}(z) .
\end{aligned}
$$

Thus $g_{j}(z) \in \mathfrak{X}_{T}\left(\mathbb{C} \backslash D_{i}\right)$ and $g_{j}\left(D_{j} \backslash D_{i}\right) \subset \mathfrak{X}_{T}\left(\mathbb{C} \backslash D_{i}\right)$.

Since the sets $D_{i}$ and $D_{j}$ are open, simply connected and $D_{j} \backslash D_{i} \neq \emptyset$, it is easy to see that $D_{j} \backslash D_{i}$ contains an accumulation point. Indeed, let $z_{0} \in D_{j} \backslash D_{i}$. If $z_{0} \notin \overline{D_{i}}$ then there is an open neighborhood of $z_{0}$ contained in $D_{j} \backslash \overline{D_{i}}$. If $z_{0} \in \partial D_{i}$, then there is a sequence $\left(z_{n}\right) \subset D_{j} \backslash D_{i}$ such that $z_{n} \rightarrow z_{0}$.

Since $\mathfrak{X}_{T}\left(\mathbb{C} \backslash D_{i}\right)$ is closed and $g_{j}\left(D_{j} \backslash D_{i}\right) \subset \mathfrak{X}_{T}\left(\mathbb{C} \backslash D_{i}\right)$, it follows that $g_{j}: D_{j} \rightarrow$ $\mathfrak{X}_{T}\left(\mathbb{C} \backslash D_{i}\right)$.

This proves (i).

(ii) is an immediate consequence of (i).

(iii) Let $z \in D_{j}$ and $x \in M \subset \mathfrak{X}_{T}\left(\mathbb{C} \backslash D_{j}\right)$. There is a function $g_{j}: D_{j} \rightarrow X$ such that $T_{D_{j}} g_{j}=x$. By (i), $g_{j}(z) \in M$ and so $x=(T-z) g_{j}(z) \in(T-z) M$.

(iv) If $\pi: X \rightarrow X / M$ is the canonical projection, then Gleason's theorem implies that the sequence $0 \rightarrow H(\Omega, M) \rightarrow H(\Omega, X) \stackrel{\pi}{\rightarrow} H(\Omega, X / M) \rightarrow 0$ is exact, [6, Prop. 2.1.5]. Thus, if $\widetilde{T}_{D_{j}} h=0$ for some $h \in H\left(D_{j}, X / M\right)$, then there exists 
$f \in H\left(D_{j}, X\right)$ such that $h=\tilde{f}$, where $\tilde{f}=\pi \circ f$. Clearly $T_{D_{j}} f \in H\left(D_{j}, M\right)$ and (iii) together with Leiterer's theorem implies that there exists $g \in H\left(D_{j}, M\right)$ such that $T_{D_{j}} f=T_{D_{j}} g$. Thus $f-g \in \operatorname{ker} T_{D_{j}} \subset H\left(D_{j}, M\right)$ by (ii). Consequently, $f \in H\left(D_{j}, M\right)$ and therefore, $h=\tilde{f}=0$.

LEMMA 2. Let $V_{1}, V_{2}$ be open subsets of $\mathbb{C}$ and suppose that $\Omega$ is an open subset of $V_{1} \cup V_{2}$. Then there exist open sets $\Omega_{1}, \Omega_{2}$ so that $\Omega_{j} \subset V_{j}, \Omega=\Omega_{1} \cup \Omega_{2}$ and an open cover $\mathcal{U}$ of $\Omega$ such that

(i) each $D \in \mathcal{U}$ is a simply connected subset of either $V_{1}$ or $V_{2}$;

(ii) if $G$ is a component of $\Omega_{1} \cap \Omega_{2}$, then there is a $D \in \mathcal{U}$ such that $D \subset G$;

(iii) $D \backslash D^{\prime} \neq \emptyset$ whenever $D, D^{\prime} \in \mathcal{U}$ are distinct.

Proof. Let $U_{j}=V_{j} \cap \Omega$ for $j=1,2$ and define $\Omega_{1}$ to be the union of all components $G$ of $U_{1}$ such that $G \backslash U_{2} \neq \emptyset$, and $\Omega_{2}$ the union of components $H$ of $U_{2}$ such that $H \backslash \Omega_{1} \neq \emptyset$. Then, each $\Omega_{j}$ is open, and every component of $\Omega_{j}$ is a component of $U_{j}$. We may assume that each $\Omega_{j}$ is nonempty. Clearly, $\Omega=\Omega_{1} \cup U_{2}$, and if $H$ is a component of $U_{2}$, then either $H \subset \Omega_{1}$ or $H \subset \Omega_{2}$. Thus $\Omega=\Omega_{1} \cup \Omega_{2}$.

Let $G_{1}, G_{2}, \ldots$ be the components of $\Omega_{1} \cap \Omega_{2}$. We note $\partial G_{n} \cap \Omega_{j} \neq \emptyset$ for each $n \in \mathbb{N}$ and $j=1$, 2. Indeed, suppose to the contrary that $\partial G_{n} \cap \Omega_{1}=\emptyset$. Let $M_{j}$ be the component of $\Omega_{j}$ containing $G_{n}$. Then $M_{1}=G_{n} \cup\left(M_{1} \backslash \overline{G_{n}}\right)$, where $G_{n} \neq \emptyset$ and where $M_{1} \backslash \overline{G_{n}}=M_{1} \backslash G_{n} \supset M_{1} \backslash M_{2} \neq \emptyset$, contradicting the fact that $M_{1}$ is connected. That $\partial G_{n} \cap \Omega_{2} \neq \varnothing$ follows similarly.

Choose $\lambda_{n} \in \partial G_{n} \cap \Omega_{1}$ and $\mu_{n} \in \partial G_{n} \cap \Omega_{2}$. Then $\lambda_{n} \notin \Omega_{2}$ and $\mu_{n} \notin \Omega_{1}$. Select $\lambda_{n}^{\prime}, \mu_{n}^{\prime} \in G_{n}$ so that $\left|\lambda_{n}-\lambda_{n}^{\prime}\right|<2^{-n}$ and $\left|\mu_{n}-\mu_{n}^{\prime}\right|<2^{-n}$. If we construct a piecewise linear path in $G_{n}$ connecting $\lambda_{n}^{\prime}$ and $\mu_{n}^{\prime}$, then, taking such a path with minimal number of segments, we obtain a path $\gamma_{n}$ between $\lambda_{n}^{\prime}$ and $\mu_{n}^{\prime}$ that does not intersect itself. Clearly it is possible to find a simply connected open set $D_{n}$ so that $\gamma_{n} \subset D_{n} \subset G_{n}$.

Let $D=\bigcup_{n} D_{n}$ and suppose that $z \in \Omega_{1} \backslash D$. We claim that there is a $\delta(z)>$ 0 such that $B(z, \delta(z)) \subset \Omega_{1}$ and $B(z, \delta(z)) \cap\left\{\mu_{1}^{\prime}, \mu_{2}^{\prime}, \ldots\right\}=\emptyset$. To this end, choose $\varepsilon(z)>0$ so that $B(z, \varepsilon(z)) \subset \Omega_{1}$, and let $n_{0}$ be such that $2^{-n_{0}}<\varepsilon(z) / 2$. Now, let $\delta(z)=\min \left\{\varepsilon(z) / 2,\left|z-\mu_{1}^{\prime}\right|, \ldots,\left|z-\mu_{n_{0}-1}^{\prime}\right|\right\}$. Then $\mu_{n}^{\prime} \notin B(z, \delta(z))$ if $n<n_{0}$, and if $n \geq n_{0}$, then $\mu_{n} \notin \Omega_{1}$ implies that $\left|z-\mu_{n}^{\prime}\right| \geq\left|z-\mu_{n}\right|-\left|\mu_{n}-\mu_{n}^{\prime}\right| \geq \varepsilon(z)-2^{-n_{0}}>\delta(z)$, as required. Similarly, if $z \in \Omega_{2} \backslash \Omega_{1}$, then there is a $\delta(z)>0$ such that $B(z, \delta(z)) \subset \Omega_{2}$ and $B(z, \delta(z)) \cap\left\{\lambda_{1}^{\prime}, \lambda_{2}^{\prime}, \ldots\right\}=\emptyset$.

We define a sequence of (possibly empty) collections of open balls recursively: for each $k \geq 1$, let $\mathcal{U}_{k}:=\left\{B\left(z, 2^{-j}\right): \delta(z) \geq 2^{-k}\right.$ and $\left.B\left(z, 2^{-k}\right) \not \subset V_{k-1}\right\}$, where $V_{0}=\emptyset$ and $V_{j}:=\bigcup_{\ell \leq j} \bigcup_{B \in \mathcal{U}_{\ell}} B$ for all $j \geq 1$. If $z \in \Omega \backslash D$, then there is a least $m$ so that $\delta(z) \geq 2^{-m}$, and so either $B\left(z, 2^{-m}\right) \subset V_{m-1}$ or $B\left(z, 2^{-m}\right) \in \mathcal{U}_{m}$. Thus $z \in V_{m}$ in either case. It follows that $\Omega \backslash D=\bigcup_{k=1}^{\infty} V_{k}=\bigcup_{\ell=1}^{\infty} \bigcup_{B \in \mathcal{U}_{\ell}} B$, and consequently $\mathcal{U}:=\left\{D_{n}\right\}_{n} \cup \bigcup_{\ell=1}^{\infty} \mathcal{U}_{\ell}$ is an open cover of $\Omega$ satisfying the desired conditions.

Lemma 3. Let $V_{1}, V_{2}$ be open subsets of $\mathbb{C}$. If $T \in B(X)$ has $(C R)$ on each $V_{j} \quad(j=$ 1, 2), then $T$ has $(C R)$ on $V_{1} \cup V_{2}$.

Proof. Let $\Omega \subset V_{1} \cup V_{2}$ be an open set. To show that $T_{\Omega}$ has closed range, let $\Omega_{1}$, $\Omega_{2}$ and $\mathcal{U}$ be as in the previous lemma, and let $f \in \overline{\operatorname{ran} T_{\Omega}}$. Since $T$ has (CR) on each $\Omega_{j}, \mathfrak{X}_{T}(\mathbb{C} \backslash D)$ is closed for each $D \in \mathcal{U}$ and there are $g_{j} \in H\left(\Omega_{j}, X\right)$ such that $\left.f\right|_{\Omega_{j}}=$ $T_{\Omega_{j}} g_{j}$ for $j=1,2$. Define $M:=\bigcap_{D \in \mathcal{U}} \mathfrak{X}_{T}(\mathbb{C} \backslash D)$. We have $T_{\Omega_{1} \cap \Omega_{2}}\left(g_{1}-g_{2}\right)=0$, and so $\left(g_{1}-g_{2}\right)\left(\Omega_{1} \cap \Omega_{2}\right) \subset M$ by Lemma 1 (ii). Thus $\left.\widetilde{g_{1}}\right|_{\Omega_{1} \cap \Omega_{2}}=\left.\widetilde{g_{2}}\right|_{\Omega_{1} \cap \Omega_{2}}$ and we can define $h \in H(\Omega, X / M)$ by $h(z)=\tilde{g}_{j}(z)$ for $z \in \Omega_{j}$. We have $\tilde{f}=\widetilde{T}_{\Omega} h$ and, again by Gleason's 
theorem, there exists $g \in H(\Omega, X)$ such that $h=\tilde{g}$. Then $f-T_{\Omega} g \in H(\Omega, M)$ and so Lemma 1 (iii) and Leiterer's theorem together imply that $f-T_{\Omega} g=T_{\Omega} k$ for some $k \in H(\Omega, M)$. Hence $f=T_{\Omega}(g+k) \in \operatorname{ran} T_{\Omega}$.

THEOREM 4. Let $T \in B(X)$. Then there is a largest open set $\rho_{C R}(T)$ on which $T$ has $(C R)$.

Proof. Let $\mathcal{W}$ be the family of all open subsets $V \subset \mathbb{C}$ such that $T$ has $(\mathrm{CR})$ on $V$. We show that $T$ has (CR) on the union $W=\bigcup \mathcal{W}$, which is obviously the largest open set on which $T$ has (CR).

Let $\Omega \subset W$ be a nonempty open subset. We show that $T_{\Omega}$ has closed range. For each $z \in \Omega$ choose $0<\delta(z)<\operatorname{dist}(z, \partial \Omega)$ so that $T$ has $(\mathrm{CR})$ on $B(z, \delta(z))$. As in the proof of Lemma 2 , define $\mathcal{U}_{k}:=\left\{B\left(z, 2^{-k}\right): \delta(z) \geq 2^{-k}\right.$ and $\left.B\left(z, 2^{-k}\right) \not \subset V_{k-1}\right\}$, where $V_{j}:=\bigcup_{\ell \leq j} \bigcup_{B \in \mathcal{U}_{\ell}} B$ and $V_{0}=\emptyset$. Then, again as in Lemma 2, $\Omega=\bigcup_{m \geq 1} V_{m}$, and so $\mathcal{U}^{\prime}:=\bigcup_{m=1}^{\infty} \mathcal{U}_{m}$ is a collection of open balls covering $\Omega$ such that $T$ has (CR) on each ball $D \in \mathcal{U}^{\prime}$ and also such that $D \neq D^{\prime}$ in $\mathcal{U}^{\prime}$ implies $D \backslash D^{\prime} \neq \emptyset$. Let $\mathcal{U}=\left(D_{k}\right)_{k \in \mathbb{N}}$ be a countable subcover of $\mathcal{U}^{\prime}$ and define $\Omega_{n}=\bigcup_{k \leq n} D_{k}$. By Lemma 3, $T$ has (CR) on each $\Omega_{n}$.

Let $M=\bigcap_{n} \mathfrak{X}_{T}\left(\mathbb{C} \backslash D_{n}\right)$. By Lemma $1, M$ is a closed subspace of $X, T M \subset M$ and $(T-z) M=M$ for all $z \in \Omega$. Denote by $\widetilde{T}: X / M \rightarrow X / M$ the operator induced by $T$ and by $\pi: X \rightarrow X / M$ the canonical projection.

Let $f \in \overline{\operatorname{ran} T_{\Omega}}$. Then for each $n$ there exists $g_{n} \in H\left(\Omega_{n}, X\right)$ such that $\left.f\right|_{\Omega_{n}}=T_{\Omega_{n}} g_{n}$. If $n \geq 2$, then $T_{\Omega_{n-1}}\left(\left.g_{n}\right|_{\Omega_{n-1}}-g_{n-1}\right)=0$ and so, by Lemma 1 (ii), $\left.g_{n}\right|_{\Omega_{n-1}}-g_{n-1}$ : $\Omega_{n-1} \rightarrow M$, i.e., $\left.\widetilde{g}_{n}\right|_{\Omega_{n-1}}=\widetilde{g}_{n-1}$ in $H\left(\Omega_{n-1}, X / M\right)$.

Define $h: \Omega \rightarrow X / M$ by $\left.h\right|_{\Omega_{n}}=\widetilde{g}_{n}$. Then $h$ is well-defined and analytic on $\Omega$. Also, $\tilde{f}=\widetilde{T}_{\Omega} h$ in $H(\Omega, X / M)$. By Gleason's theorem, there exists $g \in H(\Omega, X)$ such that $\widetilde{g}=h$ and therefore, $\pi\left(f-T_{\Omega} g\right)=0$. Exactness implies that $f-T_{\Omega} g \in H(\Omega, M)$, and so it again follows from Lemma 1 (iii) and Leiterer's theorem that there is a $k \in H(\Omega, M)$ such that $f-T_{\Omega} g=T_{\Omega} k$, i.e., $f=T_{\Omega}(g+k) \in \operatorname{ran} T_{\Omega}$.

Theorem 5. Let $T \in B(X)$ and let $V \subset \mathbb{C}$ be an open set. Suppose that the set $\{z \in V: \operatorname{ran}(T-z)$ is not closed $\}$ is countable and that, for all $z \in V$, there is an $r_{0}>0$ for which $\mathfrak{X}_{T}(\mathbb{C} \backslash B(z, r))$ is closed for all $r \in\left(0, r_{0}\right)$. Then $T$ has $(C R)$ on $V$.

Proof. Since the conditions of the theorem are inherited by every open subset $U$ of $V$, it suffices to show that $T_{V}$ has closed range in $H(V, X)$. Moreover, because the set $\{z \in \mathbb{C}: \operatorname{ran}(T-z)$ is closed and $T-z$ is not Kato $\}$ is countable by [10, II.12 Theorem 13], it follows that $E:=V \cap \sigma_{K}(T)$ is countable; let $E=\left\{\lambda_{n}: n=1,2, \ldots\right\}$ be an enumeration of $E$ (possibly finite). Note that, while $E$ need not be discrete, the set $V \backslash E=V \cap \rho_{K}(T)$ is open.

We construct a sequence $\left(B_{j}\right)$ of mutually disjoint open discs such that $E \subset \bigcup_{j} B_{j}$, $\overline{B_{j}} \subset V$ and $\mathfrak{X}_{T}\left(\mathbb{C} \backslash B_{j}\right)$ is closed for each $j$. Indeed, choose $r_{1}>0$ such that $\overline{B\left(\lambda_{1}, r_{1}\right)} \subset$ $V, \mathfrak{X}_{T}\left(\mathbb{C} \backslash B\left(\lambda_{1}, r_{1}\right)\right)$ is closed, and $\left|\lambda_{j}-\lambda_{1}\right| \neq r_{1} \quad(j \geq 2)$. Set $B_{1}=B\left(\lambda_{1}, r_{1}\right)$. Let $k$ be the smallest index such that $\lambda_{k} \notin B_{1}$ and find $r_{2}>0$ such that $B_{2}:=B\left(\lambda_{k}, r_{2}\right)$ satisfies $\overline{B_{2}} \subset V \backslash B_{1}$, the space $\mathfrak{X}_{T}\left(\mathbb{C} \backslash B_{2}\right)$ is closed and $\left|\lambda_{j}-\lambda_{k}\right| \neq r_{2} \quad(j>k)$. If we continue in this way, we obtain the required sequence of open discs $\mathcal{U}_{E}=\left(B_{j}\right)_{j}$ covering $E$.

For each $z_{0} \in V \backslash E$ we next find a simply connected open set $W_{z_{0}}$ such that $z_{0} \in W_{z_{0}} \subset V \backslash E$ and $W_{z_{0}} \backslash B_{n} \neq \emptyset$ for each $B_{n} \in \mathcal{U}_{E}$. If $z_{0} \notin \bigcup_{n} B_{n}$, choose $r>0$ such that $B\left(z_{0}, r\right) \subset V \backslash E$ and set $W_{z_{0}}=B\left(z_{0}, r\right)$. Suppose then that $z_{0} \in \bigcup_{n} B_{n} \backslash E$. Since the sets $B_{n}$ are mutually disjoint, there is only one $j$ with $z_{0} \in B_{j}$, and since the set 
$E$ is countable, there is a $\theta, 0 \leq \theta<2 \pi$ such that $\left\{z_{0}+t e^{i \theta}: t \geq 0\right\} \cap E=\emptyset$. Let $t_{0}=$ $\min \left\{t \geq 0: z_{0}+t e^{i \theta} \notin B_{j}\right\}$. Since the set $S:=\left\{z_{0}+t e^{i \theta}: 0 \leq t \leq t_{0}\right\}$ is compact and the set $E \cup \partial V$ is closed, there is an $\varepsilon>0$ such that the set $W_{z_{0}}:=\{z \in \mathbb{C}: \operatorname{dist}\{z, S\}<\varepsilon\}$ is disjoint with $E \cup \partial V$. Clearly $W_{z_{0}}$ is an open simply connected set such that $z_{0} \in W_{z_{0}} \subset$ $V \backslash E \subset \rho_{K}(T)$. If $G$ is the component of $\rho_{K}(T)$ containing $W_{z_{0}}$, then $\mathfrak{X}_{T}\left(\mathbb{C} \backslash W_{z_{0}}\right)=$ $R^{\infty}(T-\lambda)$ for every $\lambda \in G$. In particular, $\mathfrak{X}_{T}\left(\mathbb{C} \backslash W_{z_{0}}\right)$ is closed and $W_{z_{0}} \cap W_{z_{1}}=\emptyset$ if $z_{0}, z_{1} \in V \backslash E$ are such that $\mathfrak{X}_{T}\left(\mathbb{C} \backslash W_{z_{0}}\right) \neq \mathfrak{X}_{T}\left(\mathbb{C} \backslash W_{z_{1}}\right)$. By construction, $W_{z} \backslash B_{k} \neq$ $\emptyset$ and $B_{k} \backslash W_{z} \neq \emptyset$ whenever $z \in V \backslash E$ and $B_{k} \in \mathcal{U}_{E}$. Thus, if $\mathcal{U}_{K}=\left\{W_{z}: z \in V \backslash E\right\}$ and $\mathcal{U}=\mathcal{U}_{K} \cup \mathcal{U}_{E}$, then $\mathcal{U}$ is an open cover of $V$ satisfying the hypotheses of Lemma 1 .

As in Lemma 1 , let $M=\bigcap_{D \in \mathcal{U}} \mathfrak{X}_{T}(\mathbb{C} \backslash D)$ and let $\widetilde{T}: X / M \rightarrow X / M$ be the operator induced by $T$. By Lemma 1 (iii), we have $(T-z) M=M$ for all $z \in V$. We show that $\widetilde{T}-z$ is bounded below for each $z \in V \backslash E$, i.e., if $z \in V \backslash E$ and $\left(x_{n}\right)_{n} \subset X$ is such that $(\widetilde{T}-z) \tilde{x}_{n} \rightarrow 0$ in $X / M$, then $\tilde{x}_{n} \rightarrow 0$ in $X / M$.

Fix $z \in V \backslash E$ and let $x \in \operatorname{ker}(T-z)$. Then $\operatorname{ker}(T-z) \subset R^{\infty}(T-z)=\mathfrak{X}_{T}(\mathbb{C} \backslash$ $\left.W_{z}\right)$, and so there exists $g \in H\left(W_{z}, X\right)$ so that $(T-\omega) g(\omega)=x$ for all $\omega \in W_{z}$. If $h=(T-z) g$, then $h \in \operatorname{ker} T_{W_{z}}$ and, since $W_{z} \in \mathcal{U}$, it follows from Lemma 1 (ii) that $h: W_{z} \rightarrow M$. In particular, $x=h(z) \in M$. Thus $\operatorname{ker}(T-z) \subset M$.

A sequence $\left(x_{n}\right)_{n} \subset X$ satisfies $(\widetilde{T}-z) \tilde{x}_{n} \rightarrow 0$ only if there exists $\left(y_{n}\right)_{n} \subset M$ so that $(T-z) x_{n}-y_{n} \rightarrow 0$ in $X$. Since $(T-z) M=M$, there exists $\left(w_{n}\right)_{n} \subset M$ so that $(T-$ $z) w_{n}=y_{n}$ and therefore, $(T-z)\left(x_{n}-w_{n}\right) \rightarrow 0$. Since ran $(T-z)$ is closed, it follows that dist $\left(x_{n}-w_{n}, \operatorname{ker}(T-z)\right) \rightarrow 0$. $\operatorname{But} \operatorname{ker}(T-z) \subset M$, and so dist $\left(x_{n}, M\right) \rightarrow 0$, i.e., $\tilde{x}_{n} \rightarrow 0$ in $X / M$ as required. Hence $\widetilde{T}-z$ is bounded below for each $z \in V \backslash E$. In particular, $V \backslash E \subset \rho_{K}(\widetilde{T})$.

We wish to show that $\widetilde{T}_{V}$ is injective with closed range. Suppose then that $\left(f_{n}\right)_{n}$ is a sequence in $H(V, X / M)$ such that $\widetilde{T}_{V} f_{n} \rightarrow 0$. In order to show that $f_{n} \rightarrow 0$ in $H(V, X / M)$, it suffices to show that $p_{F}\left(f_{n}\right)=\sup _{z \in F}\left\|f_{n}(z)\right\| \rightarrow 0$ for every closed rectangle $F \subset V$. Suppose that $a, b, c, d$ are real numbers such that the rectangle $F=[a, b] \times[c, d] \subset V$. Choose $\delta>0$ so that $[a-\delta, b+\delta] \times[c-\delta, d+\delta] \subset V$. Since $E$ is countable, the projections $P_{1}=\{\operatorname{Re} \lambda: \lambda \in E\}$ and $P_{2}=\{\operatorname{Im} \lambda: \lambda \in E\}$ are countable and we may choose $a^{\prime}, b^{\prime} \in \mathbb{R} \backslash P_{1}$ and $c^{\prime}, d^{\prime} \in \mathbb{R} \backslash P_{2}$ so that $a-\delta<$ $a^{\prime}<a<b<b^{\prime}<b+\delta$ and $c-\delta<c^{\prime}<c<d<d^{\prime}<d+\delta$. Define $\Gamma$ to be the positively oriented boundary of the rectangle $\left[a^{\prime}, b^{\prime}\right] \times\left[c^{\prime}, d^{\prime}\right] \subset V$. Then $\Gamma \subset V \backslash E$ surrounds $F$ in the sense of Cauchy's theorem. By continuity of the minimum modulus function $z \mapsto \gamma(\widetilde{T}-z)$ on $V \backslash E$, there is a constant $c>0$ so that $\sup _{z \in \Gamma}\left\|f_{n}(z)\right\| \leq$ $c \sup _{z \in \Gamma}\left\|(\widetilde{T}-z) f_{n}(z)\right\|$ for all $n$. Thus for each $\lambda \in F$ the maximum principle implies that

$$
\left\|f_{n}(\lambda)\right\| \leq \sup _{z \in \Gamma}\left\|f_{n}(z)\right\| \leq C p_{\Gamma}\left(\widetilde{T}_{V} f_{n}\right)
$$

where $C=c|\Gamma| /(2 \pi \operatorname{dist}(\Gamma, F))$. Thus $p_{F}\left(f_{n}\right) \rightarrow 0$ as $n \rightarrow \infty$ as required. Since $(T-$ $z) M=M$ for all $z \in V$ by part (iii) of Lemma 1, Leiterer's theorem implies that $T_{V} H(V, M)=H(V, M)$, and $T_{V}$ therefore has closed range in $H(V, X)$ by [9, Prop. 2.1]; the theorem is established.

For $T \in B(X)$ denote by $K(T)$ the analytic core of $T$, i.e., the set of all $x_{0} \in X$ such that there exists a sequence $\left(x_{n}\right)_{n} \subset X$ such that $T x_{n}=x_{n-1} \quad(n \geq 1)$ and sup $\left\|x_{n}\right\|^{1 / n}<$ $\infty$. Clearly $K(T)=\bigcup_{n} \mathfrak{X}_{T}(\mathbb{C} \backslash D(0,1 / n))$. This set has been shown to play a significant role in the Fredholm theory of Banach space operators; see, for example [1]. 
Corollary 6. Let $T \in B(X)$ and let $V \subset \mathbb{C}$ be an open set. Suppose that $K(T-z)$ is closed for each $z \in V$ and that the set $\{z \in V: \operatorname{ran}(T-z)$ is not closed $\}$ is countable. Then $T$ has (CR) on $V$.

Proof. Let $z \in V$ and $K(T-z)$ be closed. Clearly $(T-z) K(T-z)=K(T-z)$ and, by the Banach open mapping theorem, there is an $\varepsilon>0$ such that $K(T-z)=$ $\mathfrak{X}_{T}(\mathbb{C} \backslash B(z, \varepsilon))$. In fact, $\varepsilon=\gamma\left(\left.(T-z)\right|_{K(T-z)}\right)^{-1}$. Clearly $\mathfrak{X}_{T}(\mathbb{C} \backslash W)=K(T-z)$ for each open set $W$ with $z \in W \subset B(z, \varepsilon)$. By Theorem 5, $T$ has (CR) on $V$.

A generalized Kato decomposition for $T \in B(X)$ is a pair of subspaces $X_{1}, X_{2} \in$ Lat $(T)$ such that $X=X_{1} \oplus X_{2},\left.T\right|_{X_{1}}$ is Kato and $\left.T\right|_{X_{2}}$ is quasinilpotent. The operator $T$ is said to be of Kato-type if $\left.T\right|_{X_{2}}$ is nilpotent. It is well known that semi-Fredholm operators are of Kato-type, see e.g. [1], [10].

If $\rho_{g k}(T)$ denotes the set of $\lambda \in \mathbb{C}$ such that $T-\lambda$ has a generalized Kato decomposition, then $\rho_{g k}(T)$ is open and $\rho_{g k}(T) \cap \sigma_{K}(T)$ accumulates only on $\partial \rho_{g k}(T)$. Indeed, suppose that $0 \in \rho_{g k}(T)$ and that $X_{1}, X_{2} \in \operatorname{Lat}(T)$ such that $X=X_{1} \oplus X_{2}$, $\left.T\right|_{X_{1}}$ is Kato and $\left.T\right|_{X_{2}}$ is quasinilpotent. If $\varepsilon>0$ is such that $B(0, \varepsilon) \subset \rho_{K}\left(\left.T\right|_{X_{1}}\right)$, then for $0<|z|<\varepsilon,(T-z) X_{2}=X_{2}$. Thus $\operatorname{ran}(T-z)=(T-z) X_{1} \oplus X_{2}$ is closed and $N^{\infty}(T-z)=N^{\infty}\left(\left.T\right|_{X_{1}}-z\right) \subset R^{\infty}\left(\left.T\right|_{X_{1}}-z\right)$.

Moreover, if $T$ has generalized Kato decomposition $\left(X_{1}, X_{2}\right)$ as above, then by the remarks preceding Lemma $1, R^{\infty}\left(\left.T\right|_{X_{1}}\right) \subseteq K(T)$. On the other hand, if $x \in K(T)$, write $x=u_{0}+v_{0}$ with $u_{0} \in X_{1}$ and $v_{0} \in X_{2}$. We show that $v_{0}=0$.

Suppose to the contrary that $v_{0} \neq 0$. Then, by definition, there are sequences $\left(u_{n}\right) \subset X_{1}$ and $\left(v_{n}\right) \subset X_{2}$ such that $T u_{n}=u_{n-1}$ and $T v_{n}=v_{n-1}$ for all $n$ and $C:=$ sup $\left\|u_{n}+v_{n}\right\|^{1 / n}<\infty$. Let $P \in B(X)$ be the projection with ker $P=X_{1}$ and $\operatorname{ran} P=X_{2}$. We have $\left\|v_{n}\right\|^{1 / n}=\left\|P\left(u_{n}+v_{n}\right)\right\|^{1 / n} \leq\|P\|^{1 / n} \cdot C$. Thus

$$
\lim _{n \rightarrow \infty}\left\|\left.T^{n}\right|_{X_{2}}\right\|^{1 / n} \geq \limsup _{n \rightarrow \infty}\left(\frac{\left\|v_{0}\right\|}{\left\|v_{n}\right\|}\right)^{1 / n}=\frac{1}{\liminf _{n \rightarrow \infty}\left\|v_{n}\right\|^{1 / n}} \geq 1 / C>0
$$

a contradiction to the assumption that $\left.T\right|_{X_{2}}$ is quasinilpotent. Hence $v_{0}=0$ and $K(T) \subseteq X_{1}$. Therefore

$$
K(T)=K\left(\left.T\right|_{X_{1}}\right)=R^{\infty}\left(\left.T\right|_{X_{1}}\right)
$$

in particular, $K(T)$ is closed.

Thus we have established the following special case of Corollary 6 , generalizing [9, Theorem 2.5].

Corollary 7. $T \in B(X)$ has $(C R)$ on $\rho_{g k}(T)$.

Duality and weak-* closed ranges. Let $\mathbb{C}_{\infty}=\mathbb{C} \cup\{\infty\}$ be the Riemann sphere and for $U$ an open neighborhood of $\infty$, let $P(U, X)$ denote the Fréchet space of analytic functions $f: U \rightarrow X$ with $f(\infty)=0$. If $T \in B(X)$, then $T$ induces a continuous mapping $T^{U}$ on $P(U, X)$ defined by $T^{U} f(z)=(T-z) f(z)+\lim _{|\omega| \rightarrow \infty} \omega f(\omega)$. For $F$ closed in $\mathbb{C}_{\infty}$ with $\infty \in F$, let $P(F, X)$ denote the inductive limit of the spaces $P(U, X)$, $U \supset F$ open; i.e., $P(F, X)$ is the $(L F)$-space consisting of germs of analytic $X$-valued functions defined in a neighborhood of $F$ and vanishing at infinity. If $\infty \in F$ is closed and $U$ is open with $F \subset U$, let $i_{U}: P(U, X) \rightarrow P(F, X)$ be defined by $i_{U} f=[f]$. Then a mapping $S$ from $P(F, X)$ to an arbitrary topological vector space $E$ is continuous if 
and only if $S \circ i_{U}$ is continuous for every open neighborhood $U$ of $F$. In particular, the mappings $T^{U}$ induce a continuous mapping $T^{F}$ on $P(F, X)$. Recall further the Grothendieck-Köthe duality principle: given $V \subset \mathbb{C}$ open, the Fréchet space $H\left(V, X^{*}\right)$ may be canonically identified with the strong dual of $P\left(\mathbb{C}_{\infty} \backslash V, X\right)$ via

$$
\langle f, g\rangle=\int_{\gamma}\langle f(z), \widetilde{g}(z)\rangle d z,
$$

where $f \in H\left(V, X^{*}\right), \widetilde{g} \in P(U, X)$ is a representative of $g \in P\left(\mathbb{C}_{\infty} \backslash V, X\right)$ and $\gamma$ is a contour surrounding $\mathbb{C} \backslash U$ in $V$. In this sense, we have that $T_{V}^{*}=\left(T^{\mathbb{C} \backslash V}\right)^{*},[6$, Theorem 2.5.12 and Lemma 2.5.13]. Moreover, by the duality results of Albrecht and Eschmeier, specifically, Theorem 21 and the proof of Theorem 5 of [2], $T^{*}$ has property $(\beta)$ on $U$ if and only if $T^{F} P(F, X)=P(F, X)$ for every closed set $F \subseteq \mathbb{C}_{\infty}$ with $\mathbb{C}_{\infty} \backslash U \subseteq F$. In this case, for every open $V \subseteq U, T_{V}^{*}$ is injective with weak-* closed range in $H\left(V, X^{*}\right)$ by a theorem of Köthe, [6, Theorem 2.5.9].

Let us say that $T^{*}$ has the property $(\mathrm{CR})^{\text {weak-* }}$ on $U$ provided that $\operatorname{ran} T_{V}^{*}$ is weak-* closed in $H\left(V, X^{*}\right)$ for every open $V \subseteq U$.

Proposition 8. Let $T \in B(X)$ and $U \subset \mathbb{C}$ open and suppose that $F$ is closed in $\mathbb{C}$ with $\mathbb{C} \backslash U \subset F$.

(i) If $T$ has $(C R)$ on $U$, then $\mathfrak{X}_{T}(F)={ }^{\perp} \mathfrak{X}_{T^{*}}^{*}(\mathbb{C} \backslash F)$, the preannihilator of $\mathfrak{X}_{T^{*}}^{*}(\mathbb{C} \backslash$ $F):=\bigcup\left\{\mathfrak{X}_{T^{*}}^{*}(K): K\right.$ compact, $\left.K \subset \mathbb{C} \backslash F\right\}$.

(ii) If $T^{*}$ has $(C R)^{\text {weak-** }}$ on $U$, then $\mathfrak{X}_{T^{*}}^{*}(F)=\mathfrak{X}_{T}(\mathbb{C} \backslash F)^{\perp}$, the annihilator of $\mathfrak{X}_{T}(\mathbb{C} \backslash$ $F):=\bigcup\left\{\mathfrak{X}_{T}(K): K\right.$ compact, $\left.K \subset \mathbb{C} \backslash F\right\}$. In particular, $\mathfrak{X}_{T^{*}}^{*}(\mathbb{C} \backslash V)$ is weak-* closed whenever $V \subseteq U$ is open.

Proof. If $F$ is closed and $\mathbb{C} \backslash U \subseteq F$, then $V:=\mathbb{C} \backslash F$ is an open subset of $U$. Thus $\operatorname{ran} T_{V}$ is closed in case (i), and $\operatorname{ran} T_{V}^{*}$ is weak-* closed in case (ii). The result now follows from parts (c) and (d) of [4, Lemma I.2.5]; alternatively, one could argue as in the proof of [6, Prop 2.5.14].

As a consequence of the Proposition 8, we obtain weak-* analogs of Theorems 4 and 5 .

THEOREM 9. There is a largest open set $V$ on which $T^{*} \in B\left(X^{*}\right)$ has $(C R)^{\text {weak-* }}$.

Proof. First we establish an analog of Lemma 3. Suppose that $T^{*} \in B\left(X^{*}\right)$ has $(\mathrm{CR})^{\text {weak-* }}$ on open sets $V_{1}$ and $V_{2}$ and that $\Omega$ is an open subset of $V_{1} \cup V_{2}$. Let $\Omega_{1} \subset V_{1} \cap \Omega, \Omega_{2} \subset V_{2} \cap \Omega$ be open sets and $\mathcal{U}$ an open cover of $\Omega$ as in Lemma 2. Let $M=\bigcap_{D \in \mathcal{U}} \mathfrak{X}_{T^{*}}^{*}(\mathbb{C} \backslash D)$. By Proposition 8, for each $D \in \mathcal{U}, \mathfrak{X}_{T^{*}}^{*}(\mathbb{C} \backslash D)$ is weak-* closed and therefore $M$ is also weak-* closed. Evidently, the restriction mapping $\left.f \mapsto f\right|_{\Omega_{j}}$ from $H\left(\Omega, X^{*}\right)$ to $H\left(\Omega_{j}, X^{*}\right)$ is weak-* continuous and intertwines $T_{\Omega}^{*}$ and $T_{\Omega_{j}}^{*}, j=1,2$. Therefore, if $f \in \overline{\operatorname{ran} T_{\Omega}^{*}}{ }^{\text {weak-* }}$, then $\left.f\right|_{\Omega_{j}} \in \overline{\operatorname{ran} T_{\Omega_{j}}^{*}}$ weak-* , and so, by assumption, there are $g_{j} \in H\left(\Omega_{j}, X^{*}\right)$ such that $\left.f\right|_{\Omega_{j}}=T_{\Omega_{j}}^{*} g_{j}$ for each $j$. As in the proof of Lemma 3, it follows from Lemma 2 that $T_{\Omega_{1} \cap \Omega_{2}}^{*}\left(g_{1}-g_{2}\right)=0$, and so $\left(g_{1}-g_{2}\right)\left(\Omega_{1} \cap \Omega_{2}\right) \subset M$ by Lemma 1 (ii). Thus $\left.\widetilde{g}_{1}\right|_{\Omega_{1} \cap \Omega_{2}}=\left.\widetilde{g}_{2}\right|_{\Omega_{1} \cap \Omega_{2}}$ in $H\left(\Omega_{1} \cap \Omega_{2}, X^{*} / M\right)$, and we can define $h \in H\left(\Omega, X^{*} / M\right)$ by $h(z)=\widetilde{g}_{j}(z)$ for $z \in \Omega_{j}$. We have $\tilde{f}=\left(T^{*} \widetilde{\tau}_{\Omega} h\right.$ and, by Gleason's theorem, there exists $g \in H\left(\Omega, X^{*}\right)$ such that $h=\widetilde{g}$. Moreover, $f-T_{\Omega}^{*} g \in H(\Omega, M)$, and so again Lemma 1 (iii) and Leiterer's theorem imply that $f-T_{\Omega}^{*} g=T_{\Omega}^{*} k$ for some $k \in H(\Omega, M)$. Hence $f=T_{\Omega}^{*}(g+k) \in \operatorname{ran} T_{\Omega}^{*}$. Thus $T^{*} \in B\left(X^{*}\right)$ has $(\mathrm{CR})^{\text {weak-* }}$ on $V_{1} \cup V_{2}$. 
To complete the argument, we adapt the proof of Theorem 4 similarly. The routine details are left to the reader.

Recall that $\operatorname{ran} T^{*}$ is weak-* closed in $X^{*}$ if and only if $\operatorname{ran} T$ is closed in $X$, [6, A.1.10]. Also, $\sigma_{K}\left(T^{*}\right)=\sigma_{K}(T),[10$, II.12 Theorem 11].

TheOrem 10. Let $T \in B(X)$ and let $V \subset \mathbb{C}$ be an open set. Suppose that the set $\{z \in V: \operatorname{ran}(T-z)$ is not closed $\}$ is countable and that, for all $z \in V$, there is a $r_{0}>0$ for which $\mathfrak{X}_{T}(\mathbb{C} \backslash B(z, r))$ is weak-* closed for all $r \in\left(0, r_{0}\right)$. Then $T^{*}$ has $(C R)^{\text {weak-* }}$ on $V$.

Proof. Since the conditions of the theorem are inherited by every open subset $U$ of $V$, it suffices to show that $T_{V}^{*}$ has weak-* closed range. Let $E:=V \cap \sigma_{K}(T)$ and construct a covering $\mathcal{U}=\mathcal{U}_{K} \cup \mathcal{U}_{E}$ exactly as in the proof of Theorem 5, noting that if $z_{0} \in V \backslash E$ and if $\lambda$ is in the component of $\rho_{K}(T)$ containing $z_{0}$, then $\mathfrak{X}_{T^{*}}^{*}\left(\mathbb{C} \backslash W_{z_{0}}\right)=$ $R^{\infty}\left(T^{*}-\lambda\right)$ is weak-* closed. Let $M=\bigcap_{D \in \mathcal{U}} \mathfrak{X}_{T^{*}}^{*}(\mathbb{C} \backslash D)$ and denote by $\left(T^{*} \tilde{)}\right.$ the operator on $X^{*} / M$ induced by $T^{*}$. Then Lemma 1 (iii) implies that $\left(T^{*}-z\right) M=M$ for all $z \in V$, and, as in the proof of Theorem $5,\left(T^{*}\right)-z$ is bounded below for each $z \in V \backslash E$. The conclusion now follows from [9, Prop. 3.1], noting that, as indicated in the proof of Theorem 5, it suffices in [9, Prop. 3.1] that the exceptional set $E$ be merely countable rather than discrete.

Corollary 11. Let $T \in B(X)$ and let $V \subset \mathbb{C}$ be an open set. Suppose that the analytic core $K\left(T^{*}-z\right)$ is weak-* closed for each $z \in V$ and that the set $\{z \in V$ : $\operatorname{ran}(T-z)$ is not closed $\}$ is countable. Then $T^{*}$ has $(C R)^{\text {weak-* }}$ on $V$. In particular, $T^{*}$ has $(C R)^{\text {weak-* }}$ on $\rho_{g k}(T)$.

Proof. The first statement follows from Theorem 10 just as Corollary 6 follows from Theorem 5. If $T \in B(X)$ has generalized Kato decomposition $\left(X_{1}, X_{2}\right)$, then $\left(X_{2}^{\perp}, X_{1}^{\perp}\right)$ is a generalized Kato decomposition for $T^{*}$ consisting of weak-* closed subspaces of $X^{*}$. Thus $\rho_{g k}(T) \subseteq \rho_{g k}\left(T^{*}\right)$. If $z \in \rho_{g k}(T)$, and $\left(X_{1}, X_{2}\right)$ is a generalized Kato decomposition for $T$, then $K\left(T^{*}-z\right)=K\left(\left.\left(T^{*}-z\right)\right|_{X_{2}^{\perp}}\right)=R^{\infty}\left(\left.\left(T^{*}-z\right)\right|_{X_{2}^{\perp}}\right)$; in particular, $K\left(T^{*}-z\right)$ is weak-* closed in $X^{*}$. Since $\rho_{g k}(T) \cap \sigma_{K}(T)$, is discrete, the last result now follows.

We are indebted to the referee and editor for their careful reading that substantially contributed to this paper.

\section{REFERENCES}

1. P. Aiena, Fredholm and local spectral theory, with applications to multipliers (Kluwer Academic Publ., Dordrecht, 2004).

2. E. Albrecht and J. Eschmeier, Analytic functional models and local spectral theory, Proc. London Math. Soc. (3) 75 (1997), 323-348.

3. E. Bishop, A duality theory for an arbitrary operator, Pacific J. Math. 9 (1959), 379-397.

4. J. Eschmeier, Analytische Dualität und Tensorprodukte in der mehrdimensionalen Spektraltheorie, Habilitationsschrift, Schriftenreihe des Mathematischen Instituts der Universität Münster, 2. Serie, Heft 42 (Münster, 1987).

5. J. Eschmeier, On the essential spectrum of Banach space operators, Proc. Edinburgh Math. Soc. (2) 43 (2000), 511-528.

6. K. B. Laursen and M. M. Neumann, An introduction to local spectral theory (Clarendon Press, Oxford, 2000). 
7. T. L. Miller and V. G. Miller, An operator satisfying Dunford's condition $(C)$ but without Bishop's property ( $\beta$ ), Glasgow Math. J. 40 (1998), 427-430.

8. T. L. Miller, V. G. Miller and M. M. Neumann, Localization in the spectral theory of operators on Banach spaces, in Proceedings of the Fourth Conference on Function Spaces at Edwardsville, Contemp. Math. 328 (Amer. Math. Soc., Providence, RI, 2003), 247-262.

9. T. L. Miller, V. G. Miller and M. M. Neumann, The Kato-type spectrum and local spectral theory, Czech. Math. J. 57 (2007), 831-842.

10. V. Müller, Spectral theory of linear operators and spectral systems in banach algebras (Birkhäuser Verlag, Basel, 2003).

11. B. Nagy, On S-decomposable operators, J. Operator Theory 2 (1979), 277-286.

12. F.-H. Vasilescu, Analytic functional calculus and spectral decompositions (Editura Academiei and D. Reidel Publishing Company, Bucharest and Dordrecht, 1982). 